\title{
Study of mutual phase locking of two gyrotrons coupled with delay
}

\author{
A.B. Adilova $^{1}$, N.M. Ryskin ${ }^{1,2}$ \\ ${ }^{1}$ Saratov State University, Saratov, Russia, adilovaab@gmail.com \\ ${ }^{2}$ Saratov Branch, Institute of Radio Engineering and Electronics, Saratov, Russia
}

\section{Introduction}

The most important application of high-power continuous-wave $(\mathrm{CW})$ gyrotrons is electron cyclotron plasma heating for nuclear fusion [1]. For such application, a large number of gyrotrons should be used to obtain a very high power. For example, in ITER it is planned to use 26 gyrotrons with $1 \mathrm{MW}$ power level. Providing coherence of radiation of large number of high-power microwave sources is a challenging task. One of the possible solutions is either injection locking by external master oscillator with stabilized frequency [2],[3] or mutual phase locking [4].

In this paper, we present the results of theoretical analysis and numerical simulation of mutual phase locking of two coupled gyrotrons.

\section{Modified quasilinear model}

In [5], we developed a basic theory of mutual phase locking of two limit-cycle oscillators coupled with delay. The delay in coupling provides essential features of the pattern of synchronization. In particular, it is very sensitive to the phase shift of the coupling signal propagating between two counterparts. However, the analysis presented in [5] is valid only in the case of weak coupling and small frequency mismatch. In [6], we considered a more general model of two coupled gyrotron oscillators, which are assumed identical except a small frequency mismatch. The model is described by two delay-differential equations (DDE)

$$
\begin{aligned}
& \frac{\mathrm{d} A_{1}}{\mathrm{~d} t}+i \frac{\Delta}{2} A_{1}+A_{1}=I_{s} \Phi\left(A_{1}\right) \cdot A_{1}+\rho e^{-i \psi} A_{2}(t-\tau), \\
& \frac{\mathrm{d} A_{2}}{\mathrm{~d} t}-i \frac{\Delta}{2} A_{2}+A_{2}=I_{s} \Phi\left(A_{2}\right) \cdot A_{2}+\rho e^{-i \psi} A_{1}(t-\tau) .
\end{aligned}
$$

Here, $A_{1,2}$ are slow amplitudes, $\Delta$ is normalized frequency mismatch, $\Phi$ is the complex gain factor, $\mathrm{I}_{s}$ is the normalized beam current parameter, $\rho$ is the coupling strength, $\tau=\omega_{0} t_{d} / 2 Q$ is the normalized delay time $t_{d}, \psi=\omega_{0} t_{d}$. In the quasi-linear theory [7], the complex gain factor is obtained as a polynomial expansion $\Phi=\alpha-\beta|A|^{2}-\gamma|A|^{4}-\ldots$ as a result of approximate solution of the electron motion equations. However, this approximation is valid only at a slight excess of the start-oscillation current and, consequently at low efficiency. Therefore, to extend the range of validity of the model, we obtain $\Phi\left(|A|^{2}\right)$ by fitting the numerical solution of the equations of time-domain gyrotron theory with fixed Gaussian profile of the RF field [7].

Numerical simulation in a wide range of parameters confirms the basic pattern of synchronization re- vealed in [5]. The results of simulation for the DDE model (1) and time-domain gyrotron theory with fixed Gaussian profile of the RF field [7] are in good agreement with each other.

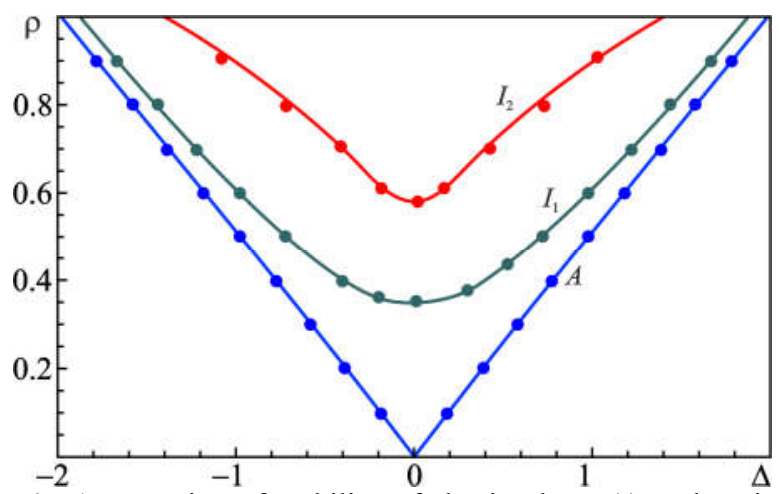

Fig. 1. Domains of stability of the in-phase $(I)$ and antiphase $(A)$ mode of synchronization on the $\Delta, \rho$ plane for $I_{s}=0.05, \psi=0.4 \pi$, and $\tau=3.0$.

The study of mutual phase locking of two gyrotrons in the soft-excitation mode $\left(\Delta_{H}=0.4\right)$ [6] revealed that the delay in coupling strongly affects the pattern of phase-locking regimes. In particular, it is very sensitive to the phase shift of the coupling signal propagating between two counterparts.

Numerical simulation in a wide range of parameters confirms the basic pattern of synchronization revealed in [5]. With the increase of the delay, there appear new modes of synchronization, i.e. the multistability becomes more pronounced. Fig. 1 illustrates the situation when there exist two in-phase and one anti-phase modes.

In addition, in Fig. 1 the stability domains calculated according the time-domain gyrotron theory with fixed Gaussian profile of the RF field [7] are shown with circles. They are in good agreement with the results of simulation for the DDE model (1).

\section{Synchronization of two gyrotrons in the hard excitation mode}

Maximum efficiency in a gyrotron is usually attained in a hard excitation mode [7]. Thus, we studied synchronization of two coupled gyrotrons with Gaussian profile of the RF field with the parameters $\mu=15$, $I_{s}=0.06, \Delta_{H}=0.53$ when the orbital efficiency is nearly $70 \%$.

The system of two mutually coupled gyrotrons demonstrates a behavior which has much common with dynamics of a single gyrotron oscillator driven by an external signal [2]. In [2], we found a number of differences from the well-studied pattern of synchronization of the generator with soft excitation. 

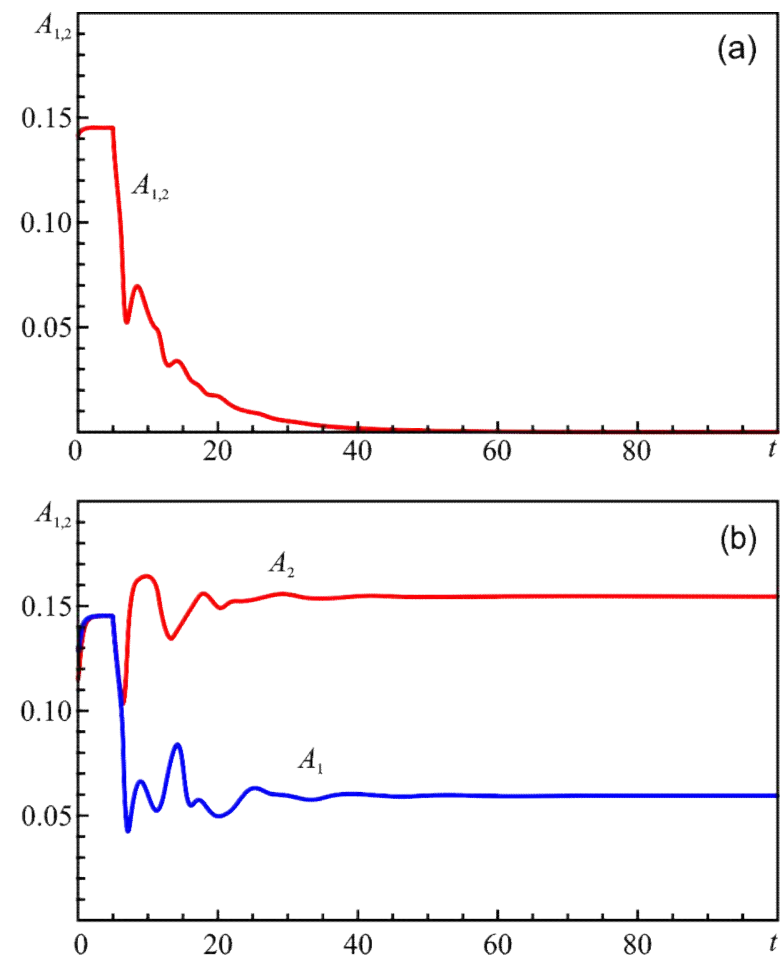

Fig. 2. Time histories of the amplitudes at $\mu=15, \Delta_{H}=0.53$, $I_{\mathrm{S}}=0.06, \rho=0.5, \psi=0.15 \pi, \tau=5$.

In particular, since bistability presents in the autonomous system, it also appears in the non-coupled system. The synchronization mode with high efficiency establishes only if the initial perturbation is large enough that is typical for systems with hard selfexcitation. Moreover, for certain values of the parameters, the synchronization mode turns out to be extremely sensitive to the initial phase of the oscillations and an external signal may cause a breakdown into the low-amplitude mode instead of synchronization. In Fig. 2, examples of the time histories for a sufficiently large coupling are presented. When both gyrotrons start from the same initial amplitudes (Fig. 2(a)), the oscillations are excited in both gyrotrons and the efficiency reaches its maximum value. However, at $t=5$ the signal from one gyrotron starts to affect the other one and vice versa. As a result, the oscillations of both gyrotrons are completely suppressed. Under somewhat different initial condition, only one of the gyrotrons suppresses the other, as is shown in Fig. 2(b).

On the contrary, coupling of the gyrotrons may lead to the excitation of one gyrotron by another. Fig. 3 illustrates the case when the initial amplitude of the second gyrotron is zero. In the hard excitation regime, this is a stable state. However, when the signal from the first gyrotron enters the resonator of the second one, the oscillation of the second gyrotron starts to grow and finally the synchronization mode is established.

\section{Synchronization of coupled multimode gyrotrons}

Finally, we considered mutual synchronization of two multimode gyrotrons. The simulation shows that in this case instead of oscillation quench there occurs excitation of a spurious mode.

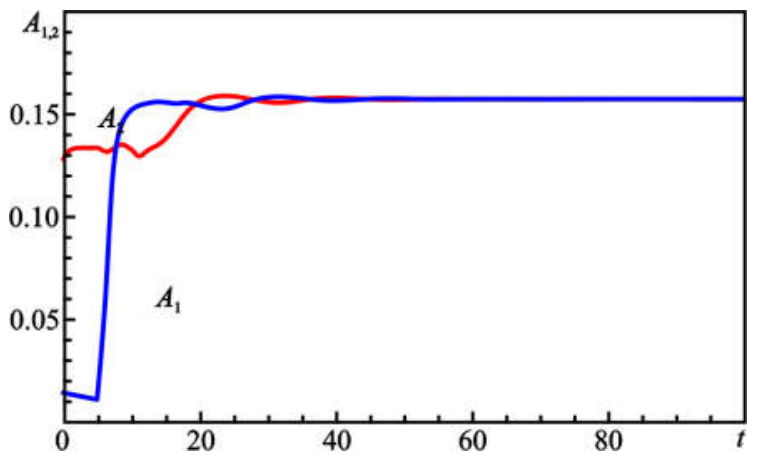

Fig. 3. Time histories of amplitudes of gyrotrons at $\rho=0.3$, $\psi=0.4 \pi, \tau=5$.

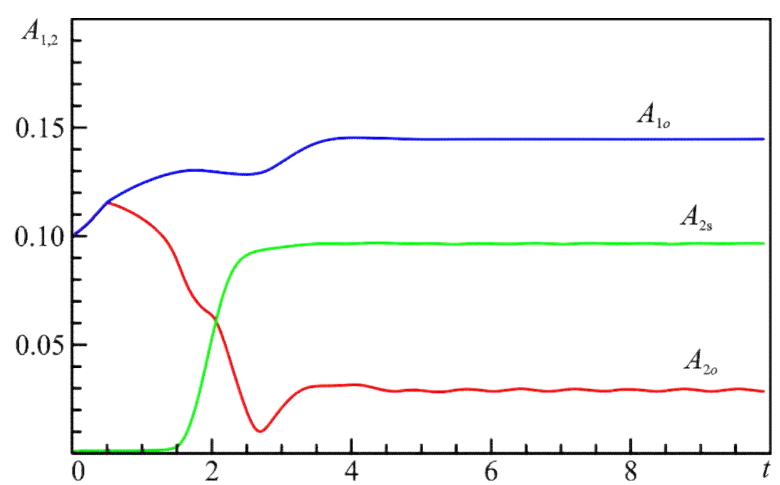

Fig. 4. Time histories of amplitudes $\left(A_{1 o}\right.$ - operation mode in the first gyrotron, $A_{2 o}$ and $A_{2 s}$ - operation and spurious modes in the second gyrotron) at $\rho=0.5, \psi=0.4 \pi, \tau=0.5$.

This is illustrated by Fig. 4 where time histories of the operating modes in two gyrotrons are plotted (cf. Fig. 2(b)). Or, on the contrary, at different initial conditions build-up of the synchronization mode may occur while the spurious modes in both gyrotrons are completely suppressed.

\section{Acknowledgment}

This work is partly supported by the Russian Foundation for Basic Research grant No. 18-0200839.

\section{References}

1. Thumm M. Recent advances in the worldwide fusion gyrotron development // IEEE Trans. Plasma Sci., 2014. V. 42, no. 3, pp. 590-599.

2. Yakunina K.A., Kuznetsov A.P., Ryskin N.M. Injection locking of an electronic maser in the hard excitation mode // Phys. Plasmas. 2015. V. 22, No 11. 113107.

3. Bakunin V.L., Denisov G.G., Novozhilova Y.V. Zones of frequency locking by an external signal in a multimode gyrotron of a megawatt power level // Radiophys. Quantum Electron., 2016. V. 58, No. 12. P. 893-904.

4. Rozental R.M., Ginzburg N.S., Glyavin M.Yu., Sergeev A.S., Zotova I.V. Mutual synchronization of weakly coupled gyrotrons // Phys. Plasmas. 2015. V. 22, No. 9. 093118.

5. Usacheva S.A., Ryskin N.M., Phase locking of two limit cycle oscillators with delay coupling // Chaos. 2014. V. 24, No. 2. 023123.

6. Adilova A.B., Gerasimova S.A., Ryskin N.M., Synchronization of delay-coupled gyrotron oscillators // EPJ Web of Conferences. 2017. V. 149. P. 04029.

7. Nusinovich G.S. Introduction to the Physics of Gyrotrons. Johns Hopkins University Press, Baltimore, 2004. 